\title{
Evolution au cours du repos hivernal de l'aptitude au débourrement, à l'allongement et à la ramification de bourgeons isolés de Douglas
}

\author{
M. Jacques ${ }^{1,2}$, M. Bonnet-Masimbert ${ }^{3}$, D. Cornu ${ }^{3}$ et E. Miginiac ${ }^{1,2}$ \\ 1 Institut de Physiologie Végétale, CNRS, 91190 Gif-sur-Yvette, \\ 2 Université P.- et-M. Curie, Laboratoire de Physiologie du Développement des Plantes, T.53-E5, 4, \\ pl. Jussieu, 75252 Paris Cedex 05, et \\ ${ }^{3}$ Station d'Amelioration des Arbres Forestiers, Ardon, 45160 Olivet, France
}

\section{Introduction}

Au cours d'une expérimentation effectuée en conditions contrôlées, des Douglas (Pseudotsuga menziesii (Mirb.) Franco) jeunes issus de semis, traités par des moyens divers de levée de repos hivernal, avaient montré des capacités de débourrement et de croissance qui variaient selon les conditions de l'environnement (Pivert et al., 1986). L'éclairement se comportait comme un déterminant majeur du développement des plantes. En même temps, une hétérogénéité dans l'état physiologique des bourgeons des jeunes arbres avait été observée.

Le présent travail a pour but d'étudier l'influence de l'éclairement sur les capacités de développement de bourgeons de Douglas au cours de leur phase de dormance en conditions naturelles. Afin de soustraire les bourgeons aux influences corrélatives et de disposer d'un matériel abondant et homogène, une technique de bouturage d'extrémités de rameaux prélevées sur 2 clones de Douglas, analogue à la technique de boutures de nœuds utilisée chez les feuillus (Mauget et Germain, 1980) a été mise en œuvre.

\section{Matériel et Méthodes}

Les extrémités de rameaux sont prélevées chaque mois, d'octobre à avril, sur 2 clones de Douglas (1800 et 1798), cultivés sous forme de haie depuis 1977 à la station d'Amélioration des Arbres Forestiers d'Orléans. Ces extrémités de rameaux de $5 \mathrm{~cm}$, dont seul le bourgeon terminal est conservé, sont placées dans des enceintes climatisées du Phytotron de Gif-surYvette, sur du sable humide à $22^{\circ} \mathrm{C}, 16 \mathrm{~h}$ d'éclairement et $70 \%$ d'humidité relative, où leur évolution est suivie. A chaque prélèvement, 20 bourgeons sont maintenus intacts tandis que 20 autres sont totalement débarrassés de leurs écailles; seules les écailles brunes sont supprimées sur 20 autres bourgeons. L'expérience a lieu sous 2 éclairements, 250 et 450 $\mu \mathrm{E} \cdot \mathrm{m}^{-2} \cdot \mathrm{s}^{-1}$. 


\section{Résultats}

Sauf mention spéciale, seuls les résultats concernant le clone 1800 dont les réactions sont plus marquées, seront détaillés.

\section{Débourrement et caractéristiques de l'évolution des pousses}

Pour les prélèvements d'octobre à décembre, aucun des bourgeons intacts ne débourre; la majorité des bourgeons sans écailles ou avec écailles blanches, après un bref gonflement, reconstituent en une quinzaine de jours, un bourgeon écailleux par transformation des ébauches des aiguilles les plus basales de l'apex dénudé en écailles, ou des écailles blanches en écailles brunes. Ce dernier type d'évolution est qualifié de "transformation directe"; il devient de moins en moins fréquent au cours du temps. Pour les prélèvements réalisés à partir de janvier, les bourgeons intacts débourrent plus nombreux et plus vite jusqu'en avril où le débourrement est total.
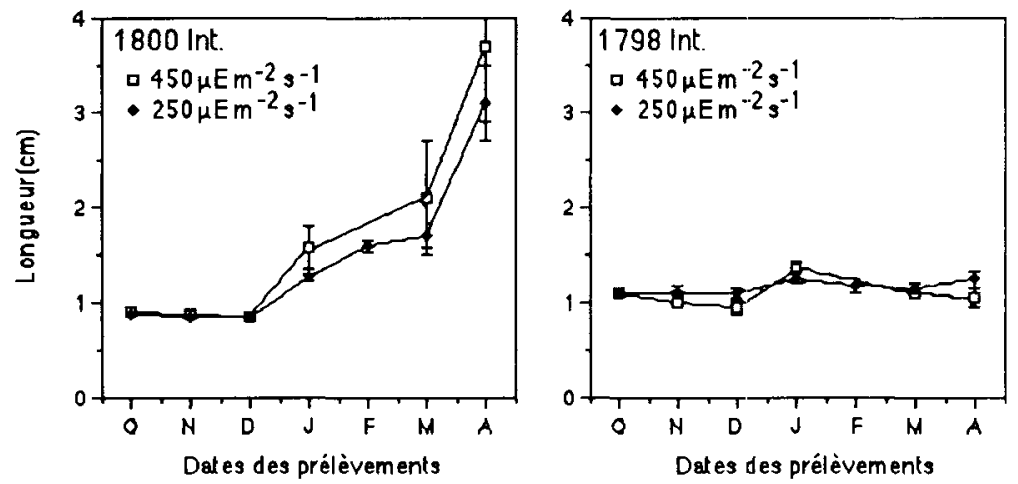

Fig. 1. Allongement maximal de bourgeons, isolés, intacts (Int.) de 2 clones de Douglas, 1798 et 1800 , prélevés au cours de l'arrêt de croissance, placés sur du sable humide, à $22^{\circ} \mathrm{C}$, en 16 h d'éclairement de 250 et 450 $\mu \mathrm{E} \cdot \mathrm{m}^{-2} \cdot \mathrm{s}^{-1}$. $\mathrm{l}$ : écart-type.
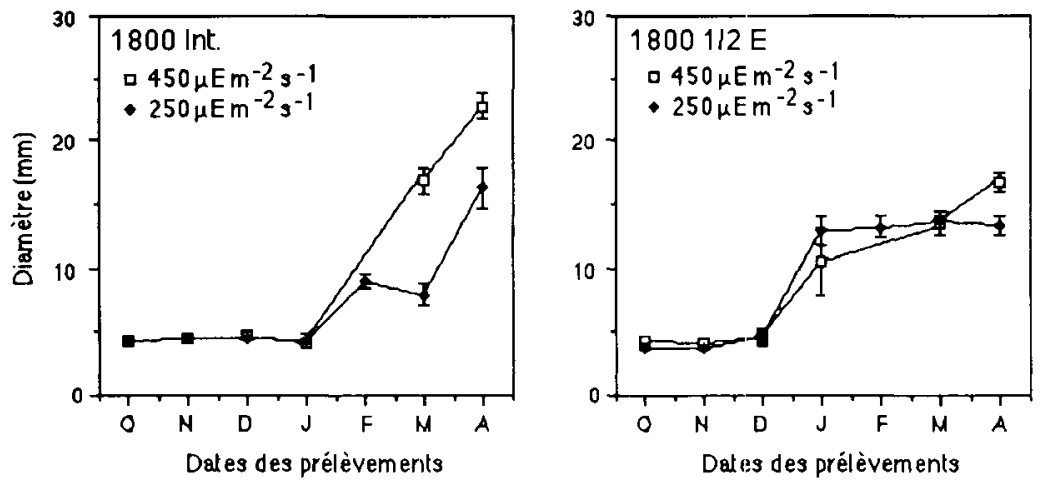

Fig. 2. Diamètre maximal de bourgeons isolés intacts (lnt.) ou dépourvus de leurs écailles brunes (1/2 E) du clone 1800 de Douglas, prélevés au cours de l'arrêt de croissance, placés sur du sable humide, à $22^{\circ} \mathrm{C}$, en $16 \mathrm{~h}$ d'éclairement de 250 et $450 \mu \mathrm{E} \cdot \mathrm{m}^{-2} \cdot \mathrm{s}^{-1}$. I: écart-type. 

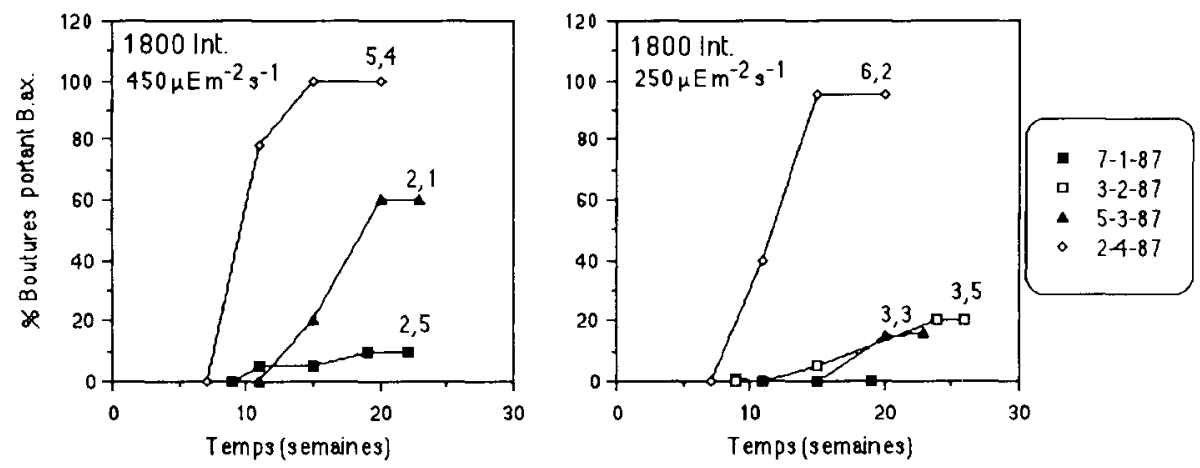

Fig. 3. Aptitude à la ramification de bourgeons isolés intacts du clone 1800 de Douglas, prélevés à différents moments (voir encadré) au cours de larrêt de croissance, placés sur du sable humide, à $22^{\circ} \mathrm{C}$, en $16 \mathrm{~h}$ d'éclairement de 250 et $450 \mu \mathrm{E} \cdot \mathrm{m}^{-2} \cdot \mathrm{s}^{-1}$. Le zéro des abscisses correspond à la date du prélèvement; les nombres représentent les nombres maximaux moyens de bourgeons axillaires par bouture.

A la fin des 6 à 8 mois d'observation, les bourgeons terminaux atteignent des niveaux d'évolution d'autant plus marquée que le prélèvement du rameau a été plus tardif. Cette évolution va du bourgeon d'origine non débourré au bourgeon dont les écailles brunes sont seulement écartées laissant apparaître les écailles blanches, puis l'élongation de la nouvelle tige se termine par un bourgeon encore vert et plus généralement par un bourgeon écailleux, brun. Le stade bourgeon brun est acquis plus rapidement par les bourgeons écaillés que par les bourgeons intacts.

Le clone 1798 a, tout au long de l'année, une propension au débourrement. Dès les premiers prélèvements d'octobre des écailles blanches apparaissent. En revanche, son allongement est toujours limité (Fig. 1).

\section{Longueur des pousses formées}

A partir du mois de janvier, les bourgeons intacts (Fig. 1) donnent naissance à des pousses d'autant plus longues que l'on se rapproche de la date du débourrement naturel. L'éclairement fort favorise l'élon- gation. Les pousses issues de bourgeons écaillés ont un allongement inférieur à celui des pousses de bourgeons intacts et la longueur finale est atteinte plus vite, par suite de la transformation rapide du méristème terminal en bourgeon écailleux.

\section{Expansion du bourgeon et des aiguilles}

La mesure du diamètre maximal du bourgeon ou de la pousse avec ses aiguilles rend compte du gonflement du bourgeon et du degré d'expansion des aiguilles. Ainsi, au cours du mois de janvier les bourgeons intacts s'allongent légèrement mais ne présentent aucune expansion des aiguilles. Cette expansion est favorisée par l'éclairement et est plus importante pour les prélèvements les plus tardifs (Fig. 2).

\section{Aptitude à la ramification}

Les nouvelles pousses ont un nombre d'aisselles porteuses de bourgeons axillaires d'autant plus grand que les rameaux d'origine ont été prélevés plus tardivement. 
L'influence de la valeur de l'éclairement est très marquée sur ce phénomène. Ainsi pour le prélèvement du mois de mars l'apparition des bourgeons axillaires est plus intense et plus précoce sur les pousses placées en éclairement fort. Au mois d'avril le niveau d'éclairement n'intervient plus (Fig. 3).

Pour le clone 1798, l'aptitude à la ramification est semblable mais l'intensité de cette dernière est plus faible que pour le clone 1800.

\section{Discussion et Conclusion}

Les bourgeons isolés de Douglas se comportent d'une façon analogue à celle des plants entiers étudiés par Campbell et Sugano (1975); leur développement est d'autant plus avancé que les plantes sur lesquelles ils ont été prélevés ont séjourné plus longtemps à l'extérieur où les températures froides et les jours en cours d'allongement conjuguent leur action pour lever la dormance.

La valeur de l'éclairement subi depuis le prélèvement a une influence sur les possibilités du débourrement, sur la croissance et l'initiation des bourgeons axillaires.

L'influence des écailles est double. La suppression des écailles, totale ou partielle, provoque pour les bourgeons qui, à l'état écailleux, ne débourrent pas spontanément une certaine croissance. Pour des bourgeons capables de croître, cette suppression entraîne un retour plus rapide à l'état écailleux et une élongation moindre.

L'importance du rôle de l'éclairement qui parvient au méristème au cours de l'initiation des organes reproducteurs a été soulignée chez 2 Pinus par Kosinski et Giertych (1982). Il est probable que les écailles ont ici un rôle de filtre de l'éclairement. Quant l'éclairement fort atteint l'apex, d'une part son développement est accéléré et intensifié, d'autre part ses capacités de ramifications sont modifiées, l'initiation des bourgeons axillaires se produisant naturellement à l'intérieur du bourgeon écailleux dans les semaines qui précédent le débourrement (Owens, 1969). Les écailles pourraient aussi agir comme source d'inhibiteurs de croissance comme chez le pommier (Swartz et al., 1984). Toutefois, il faut noter qu'un essai préliminaire avait montré que le développement des bourgeons écaillés est rapidement arrêté à l'obscurité, ce qui confirme l'influence primorcliale de l'éclairement et que les écailles doivent intervenir en tant que filtre de la lumière.

Le clone 1798 se caractérise par sa propension au débourrement (quelques bourgeons intacts présentent un écartement des écailles dès le mois d'octobre) mais sa croissance est faible dans nos conditions expérimentales. Le clone 1800 a des réactions de croissance et de développement plus marquées en fonction de la date des prélèvements; il a des exigences plus stricts pour son débourrement.

La quantification de l'intensité de la dormance de bourgeons de feuillus est essentiellement basée sur la mesure du délai moyen de débourrement des bourgeons de boutures de nœuds; un certain nombre d'auteurs, dont Rageau, qui en 1978 avait mesuré l'allongement de feuillus, ont considéré que ce repère était imparfait car il ne tient pas compte des capacités réelles de croissance des bourgeons. La technique employée ici permet de caractériser l'état physiologique d'un bourgeon, non seulement par ses capacités de débourrement mais aussi par ses possibilités de croissance et de ramification ultérieure; elle pourrait constituer un test de dormance pour les conifères. 


\section{Références}

Campbeil R.K. \& Sugano A.l. (1975) Phenology of bud in Douglas fir related to provenance photoperiod, chilling flushing temperature. Bot. Gaz. 136, 290-298

Kosinski G. \& Giertych M. (1982) Light conditions inside developing buds affect floral induction. Planta 155, 93-94

Mauget J.C. \& Germain E. (1980) Dormance et précocité de débourrement des bourgeons chez quelques cultivars de noyer (Juglans regia L.). C.R. Acad. Sci. Paris Ser. D 290, 135-138

Owens J.N. (1969) The relative importance of initiation and early development on cone pro- duction in Douglas fir. Can. J. Bot. 47, 1039 1049

Pivert F., Jacques M., Bonnet-Masimbert M. \& Miginiac E. (1986) Comportement des bourgeons de Pseudotsuga menziesii (Mirb.) Franco cultivés en conditions contrôlées. Ann. Sci. For. 43, 521-532

Rageau R. (1978) Croissance et débourrement des bourgeons végétatifs du pêcher (Prunus persica L. Batsch) au cours d'un test classique de dormance. C.R. Acad. Sci. Paris Ser. D 287, 1119-1122

Swartz H.J., Geyer A.S., Powell L.E. \& Lin S.H.C. (1984) The role of bud-scales in the dormancy of apples. J. Am. Soc. Hortic. Sci. 109, 745-749 